\title{
An approach to antibiotic treatment in patients with sepsis
}

\author{
María Luisa Martínez ${ }^{1}$, Erika P. Plata-Menchaca ${ }^{2}$, Juan Carlos Ruiz-Rodríguez ${ }^{2,3}$, Ricard Ferrer ${ }^{2,3,4}$ \\ ${ }^{1}$ Department of Intensive Care, Hospital Universitario General de Catalunya, Barcelona, Spain; ${ }^{2}$ Shock, Organ Dysfunction, and Resuscitation \\ Research Group, Vall d'Hebron Research Institute, Barcelona, Spain; ${ }^{3}$ Department of Intensive Care, Vall d'Hebron University Hospital, Barcelona, \\ Spain; ${ }^{4}$ Centro de Investigación Biomédica en Red (CIBER) de Enfermedades Respiratorias, Barcelona, Spain \\ Contributions: (I) Conception and design: All authors; (II) Administrative support: R Ferrer; (III) Provision of study materials or patients: JC Ruiz- \\ Rodríguez, ML Martínez, EP Plata-Menchaca; (IV) Collection and assembly of data: ML Martínez, R Ferrer; (V) Data analysis and interpretation: \\ All authors; (VI) Manuscript writing: All authors; (VII) Final approval of manuscript: All authors. \\ Correspondence to: Ricard Ferrer. Department of Intensive Care, Vall d'Hebron University Hospital, Passeig de Vall d'Hebron 119-129, 08035 \\ Barcelona, Spain. Email: r.ferrer@vhebron.net.
}

\begin{abstract}
Sepsis is a medical emergency and life-threatening condition due to a dysregulated host response to infection, which is time-dependent and associated with unacceptably high mortality. Thus, when treating suspicious or confirmed cases of sepsis, clinicians must initiate broad-spectrum antimicrobials within the first hour of diagnosis. Optimizing antibiotic use is essential to ensure successful outcomes and to reduce adverse antibiotic effects, as well as preventing drug resistance. All likely pathogens involved should be considered to provide an appropriate antibiotic coverage. Clinicians must investigate on the previous risk of multidrug-resistant (MDR) pathogens, and the principle of individualized dosing should replace the principle of standard dosing. The loading dose is an initial higher dose of an antibiotic for all patients, yet an individualized treatment approach for further doses should be implemented according to pharmacokinetics $(\mathrm{PK})$ /pharmacodynamics (PD) and the presence of renal/liver dysfunction. Extended or continuous infusion of beta-lactams and therapeutic drug monitoring (TDM) can help to achieve therapeutic levels of antimicrobials. Reevaluation of duration and appropriateness of treatment at regular intervals are also necessary. De-escalation and shortened courses of antimicrobials must be considered for most patients, except in some justified circumstances. Leadership, teamwork, antimicrobial stewardship (AS) frameworks, guideline's recommendations on the optimal duration of treatments, de-escalation, and novel diagnostic stewardship approaches will help us to improve patients' quality of care.
\end{abstract}

Keywords: Sepsis; septic shock; antimicrobial therapy; antimicrobial stewardship (AS); timing; de-escalation; early antibiotics; early antimicrobial therapy in sepsis; sepsis treatment

Submitted Dec 19, 2019. Accepted for publication Jan 08, 2020.

doi: $10.21037 /$ jtd.2020.01.47

View this article at: http://dx.doi.org/10.21037/jtd.2020.01.47

\section{Introduction}

Sepsis is a medical emergency and a significant public health problem that affects millions of people worldwide, representing one of the leading causes of death (1). In sepsis, a dysregulated host response to infection leads to a potentially life-threatening condition caused by organ dysfunction (2). In addition, the reported incidence of sepsis is increasing, probably reflecting population aging, potentially being affected by more comorbidities, and better recognition of the condition (3).

Despite significant advances in our understanding of sepsis pathophysiology, numerous clinical trials have failed to identify new therapies capable of modifying the course of the disease $(4,5)$. It is of prime importance to acknowledge sepsis as a medical emergency as, in the absence of any definitive treatment, successful interventions involve the timely control of infection and organ support (6). The 2016 Surviving Sepsis Campaign (SCC) guidelines strongly recommend that the administration of intravenous broad- 


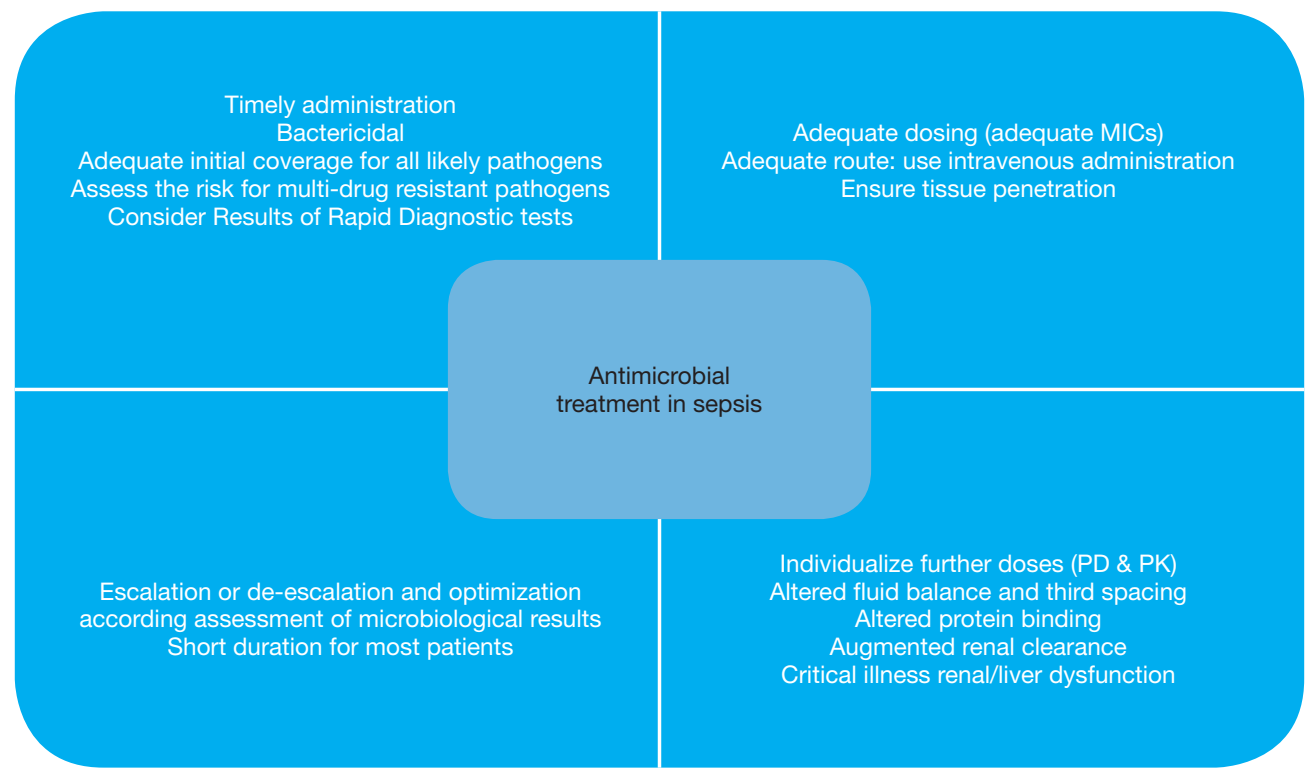

Figure 1 Practice peals for antibiotic treatment in sepsis. MICs, minimum inhibitory concentrations. PD, pharmacodynamics; PK, pharmacokinetics.

spectrum antibiotics should be initiated as soon as possible, preferably within an hour of sepsis recognition $(7,8)$.

Several studies on sepsis and septic shock have shown that delayed administration of antibiotics is associated with detrimental outcomes (9-12). Beyond its apparent benefits, broad-spectrum antibiotics can cause considerable harm, including antibiotic-associated adverse events and life-threatening complications due to antimicrobial resistance (13-16).

The number of infections due to multidrug-resistant (MDR) pathogens has dramatically increased worldwide, thus limiting our therapeutic arsenal. Some estimates suggest that antimicrobial resistance will be responsible for around ten million deaths annually by 2050 (17). Therefore, one of the main concerns on the management of patients with sepsis and septic shock is the augmentation of antimicrobial efficacy, while preventing the emergence of resistant strains during treatment. In this context, antimicrobial stewardship (AS) represents a meaningful strategy for sepsis management, as it involves an indepth focus on multi-professional collaboration, as well as the appropriateness, adequacy, and optimization of antimicrobial therapy (18). Interdisciplinary AS teams (e.g., including infectious disease specialists, pharmacists, microbiologists) are becoming a standard of care for patients with sepsis. AS has been defined as "the optimal selection, dosage, and duration of antimicrobial treatment that results in the best clinical outcome for the treatment or prevention of infection, with minimal toxicity to the patient and minimal impact in subsequent resistance" (19). The best antimicrobial therapy was defined by Joseph and Rodvold as the four D's: "right drug, right dose, de-escalation, and right duration" (20).

Antimicrobial pharmacokinetics (PK) and pharmacodynamics (PD) are also important considerations to improve treatment success. In critically ill patients, the individualized dosing approach should replace the standardized dosing approach. AS also implies de-escalation (switching to or interruption of a drug class resulting in a less broad spectrum of coverage) and shortening the duration of treatment (Figure 1).

In this review, the authors gathered the most relevant evidence regarding antimicrobial management in sepsis, focusing on timing, empiric antimicrobial selection, optimal dosing, de-escalation, duration, and, accordingly, the importance of implementing AS policies.

\section{Timing of antimicrobial therapy}

The central concept to keep in mind while reading this paper is that sepsis is a severe and time-dependent condition. Both the early administration of appropriate antimicrobial therapy and effective source control are critical 
interventions to improve survival. In recent years, highquality evidence has demonstrated that protocolized care and early resuscitation in sepsis are the best approaches to reduce deaths (21-23). The 2016 SSC guidelines established a set of recommendations or bundles, including the early administration of intravenous broad-spectrum antimicrobial agents within 1 hour or, even sooner, in patients with sepsis and septic shock $(5,6)$. Different scientific societies give support for the importance of timing, as different studies have pointed out the potential benefits of the early initiation of appropriate antibiotic therapy $(7,19,22,23)$. Recent evidence has demonstrated the association between bundle compliance and improved survival of patients with sepsis and septic shock. As a consequence, different medical organizations in the United States have adopted the recommendations of the 2016 SSC as mandatory policies $(8,24,25)$. However, we position against any quality metric or reimbursement policy that mandates slavish adherence to a particular recommendation of the 2016 SSC guidelines, as the implementation of available clinical tools should not substitute clinical judgment (26).

There are many studies on the effects of delayed administration of antibiotics in patients with sepsis and septic shock (27-32). The first major analysis was conducted in 2006 by Kumar et al. In their study, the authors performed a retrospective analysis of 2,154 patients, showing that administration of effective antimicrobial therapy within the first hour of appearance of hypotension was associated with the highest survival probability. The survival rate dropped an average of $7.6 \%$ for each hour of antimicrobial delay after hypotension onset (11). In a retrospective analysis of 261 patients with sepsis and septic shock, Gaieski et al. demonstrated that antibiotics significantly decreased mortality when they were given within $\leq 1 v s .>1$ hour from triage and qualification for early-goal directed therapy (33). In a prospective study of 291 patients with septic shock in the emergency department (ED), Puskarich et al. found that delayed administration of antibiotics was associated with increased mortality (34). In a systematic review, Sherwin $e t$ al. reported that administration antimicrobial therapy within one hour of shock recognition had the most significant benefit for reducing mortality (35). Some studies have also found an association between longer elapsed times to antimicrobial administration and worse outcomes, even in a linear way $(9,10,36-40)$. In a previous retrospective study of 17,990 patients in 165 intensive care units (ICU), Ferrer et al. showed that in-hospital mortality regularly increased with hourly delays in antibiotic administration (9). The negative impact of delays was demonstrated, independently of the severity of disease or hospital admission area. In a clusterrandomized trial, the Medical Education for Sepsis Source Control and Antibiotics (MEDUSA) study group evaluated the effect of a multifaceted educational intervention to improve antibiotic treatment on sepsis mortality. The authors found a $2 \%$ increase in mortality for every hour of antibiotic delay in more than 4,000 patients with sepsis and septic shock (41). In 2017, in a large retrospective and multicenter study of 35,000 patients with sepsis admitted to the ED, Liu et al. reported that each elapsed hour between presentation and antibiotic administration was associated with a $9 \%$ increase in the mortality risk (10). The most significant benefit of early antibiotic administration was observed in patients with septic shock. In contrast to these findings, in the Puskarich et al.'s study (34), the authors failed to demonstrate a linear relationship between hourly delays from ED triage to treatment and mortality.

The New York State Department of Health recently reported the first record after the implementation of a statewide mandate requiring protocolized sepsis care (36). Among 49,331 patients at 149 hospitals, 40,696 (82.5\%) had the 3-hour bundle completed within 3 hours. Among patients who had the 3-hour bundle completed within 12 hours, an extended time to the initiation of antibiotics was associated with higher risk-adjusted in-hospital mortality (OR, 1.04 per hour; 95\% CI, 1.03-1.06; $\mathrm{P}<0.001$ ); the linear associations seemed to be stronger in patients with shock. Peltan et al. published a retrospective study of 10,811 ED patients with sepsis who were admitted to four hospitals (40). After adjustment, each additional hour from ED arrival to the first dose of antibiotics was associated with a $10 \%(95 \%$ CI, $5-14 \%$; $\mathrm{P}<0.001)$ increased odds of 1 -year mortality.

Not only has delayed administration of antibiotics been associated with increased mortality, but also other adverse consequences. Increased patient's length of stay $(32,42)$, acute lung injury (43), acute kidney injury (44), and worsening organ dysfunction due to an exacerbated inflammatory response are worrisome $(45,46)$. In contrast with these results, other studies have failed to demonstrate a benefit of early antimicrobial therapy (47-51). In a prior meta-analysis of 11 studies, including data from $>16,000$ patients with sepsis and septic shock, Sterling et al. found a non-significant difference in mortality when comparing the administration of antibiotics within 3 hours from ED triage, and within 1 hour from shock recognition (52). This meta-analysis was questioned due to high heterogeneity. In a more recent meta-analysis that included 11 studies of patients with sepsis who arrived to the 
ED (53), Kalil et al., found a significant reduction of 33\% in mortality when comparing immediate (within 1 hour) and delayed ( $>1$ hour) antibiotic administration [OR, 0.67 (95\% CI, 0.59-0.75)]. In a recent systematic review, Sherwin et al. concluded that patients with sepsis and septic shock should receive early and appropriate antibiotics during ED admission. The highest reduction in mortality was observed in patients with septic shock who received appropriate antimicrobial therapy within 1 hour from sepsis recognition (35). A randomized controlled open-label trial of 2,672 patients with suspected sepsis first compared the effects of early pre-hospital antibiotics and ED antibiotics (51). In this study, the authors found that giving pre-hospital antibiotics shortened the time to the first antibiotic dose by 96 minutes, but did not improve mortality. The limitation of this study was the low mortality rate reported (8\%), given that more than $95 \%$ of patients had an infection without sepsis or septic shock criteria. Of note, this study suggests that clinicians should gather additional data when facing infections without sepsis/septic shock criteria before prescribing antimicrobials.

Despite mixed results, a substantial agreement exists among international experts regarding the establishment of earlier antimicrobial therapy in septic shock, and novel concepts have been recently introduced. The "doorto-needle" time of $60 \mathrm{~min}$ for antibiotic administration has been proposed, reflecting global concerns regarding establishing a time window for effective treatment after sepsis recognition $(13,54)$. Nevertheless, achieving an effective application of institutional protocols administering antimicrobials within 1 hour from presentation remains challenging. Immediate medication availability, general beliefs regarding sepsis severity and strict local policies hamper initiatives aimed to encourage improvements in quality of care. Moreover, according to the Infectious Diseases Society of America (IDSA), stipulating an aggressive, fixed-time period for antibiotic administration may lead to deleterious consequences (55). Singer questioned the "each hour delay" statement that promotes clinicians' compliance to time goals for antibiotic administration, based on the risks associated with increasing antimicrobial resistance and the probability of prescribing broad-spectrum antibiotics in patients with sepsis-like syndromes (13). Most experts and scientific societies agree that if an infection cannot be ruled out in a patient with acute organ failure or shock, it is understandable and appropriate to administer broad-spectrum antibiotics as soon as possible. Instead, if a patient presents with less severe disease and an alternative diagnosis is most probable than infection, then a reasonable approach should be gathering additional diagnostic data before administering broad-spectrum antibiotics $(14,15,56-58)$.

\section{How to achieve the goal of timely antibiotic administration}

The main factors determining the success of antimicrobial therapy in sepsis are both the early recognition of severe infection and timely administration of appropriate antibiotic therapy. For clinicians, the decision to attribute organ dysfunction to infection is challenging and requires the combination of vast clinical knowledge and competent clinical judgment. According to the sepsis-3 definition criteria (2), the Sequential Organ Failure Assessment (SOFA) score is a useful tool to assess organ dysfunction. A simplified score, the quick SOFA (q-SOFA) sore, can also be applied to identify patients with infection and a high risk of death (59).

Recently, Filbin et al. published a retrospective analysis of adult patients with sepsis to address the feasibility of achieving the 1-hour goal from ED triage to antibiotic administration (60). This study showed that unspecific symptoms at presentation, transfer from triage to nonacute areas, and lower SOFA scores were the factors independently associated with antibiotic delays. Other studies have also analyzed patient-related factors and internal policies interfering with the early administration of antibiotics (61-63). In a previous study, Peltan et al. showed that antibiotics were administered earlier in patients who received pre-hospital emergency care and transferred to the $\mathrm{ED}$, in contrast to patients being transferred from medical wards to the ED (62). In a recent prospective pre/ post quasi-experimental study conducted in a single ED, Petit et al. found that the q-SOFA score at triage was poorly sensitive for early sepsis detection, and did not improve antibiotic timing or outcomes in patients admitted with suspected bacterial infection (64). Antibiotics were more frequently prescribed in patients with a q-SOFA $\geq 2$ than those patients with a q-SOFA $<2$. In addition, earlier administration of antibiotics was observed in patients with a q-SOFA $\geq 2$, when comparing both groups. The effects of implementing the sepsis-3 criteria on improving the early detection and timely management of sepsis remain to be seen. Shortly, novel technologies will improve the time to diagnosis and optimal treatment selection for patients with suspected sepsis.

The possible solutions for preventing antibiotic delays 
may include multi-professional (e.g., critical care physicians, nurses, pharmacists, microbiologists, administrators), cooperative and developing programs for improving sepsis management. Educational and training programs aimed to refine sepsis awareness and management have improved the suitability of antimicrobial therapy in sepsis $(51,65)$. The use of automated electronic sepsis alert systems and improving communication among hospital staff are also beneficial (66-68). Hospital administrative structures and alignments must be adapted to facilitate the performance of crucial treatment interventions regardless of the patient's location and, preferably, before patients are planned to be transferred to the ICU (69). In this sense, the implementation of an institutional "sepsis code" protocol has attained excellent results (70). Adequate antibiotic dispensing and immediate availability of the most frequently used antibiotics should be part of a pharmacy's diligences (71). The availability of premixed antimicrobial preparations was evaluated to reduce the time to administration of the first antimicrobial agent in the ED (72).

\section{Appropriateness of empiric antimicrobial therapy}

The prescription of an empiric antibiotic treatment targeting the most probable pathogens involved is an essential step to improve patient outcomes. There is a broad literature on the detrimental impact and adverse outcomes of inappropriate empiric therapy in sepsis $(11,18,35,69,73-76)$. Therefore, the 2016 SSC guidelines recommend that the initial treatment should include a broad-spectrum antibiotic (alone or in combination) that has activity against all likely pathogens (7). Of note, the prescription of an inadequate empiric therapy is prevailing, and occurs in $10 \%$ to $40 \%$ of cases, depending on the incidence of MDR pathogens and the degree of adherence to clinical guidelines $(65,75-78)$. The coexistence of delays and inadequacy of treatment have been associated with lower survival rates.

Recent studies have shown that the best benefit from adequate antibiotic treatment is obtained in patients with higher severity-of-illness scores. Kumar et al. reported that inappropriate empiric antimicrobial therapy was associated with a 5 -fold reduction in survival (from $55 \%$ to about $11 \%$ ), in over 5,000 patients with septic shock (12). In a prospective study of patients with septic shock and high severity scores, with an average of three dysfunctional organs, Suberviola Cañas et al. found that patients who received inadequate antibiotics had significantly higher mortality rates compared with the patients who received appropriate treatment (33.8\% vs. $51.2 \% ; \mathrm{P}=0.03$ ) (74). In an interesting retrospective analysis of 2,594 patients, VazquezGuillamet et al. found that the number needed to treat with appropriate antimicrobial therapy to prevent one patient death was 4. All patients had severe sepsis or septic shock and positive blood cultures to assess the appropriateness of antimicrobial therapy (76).

Selecting an adequate empiric antimicrobial therapy involves covering all likely microorganisms causing the suspected source of infection. Patient- and pathogenrelated factors need to be considered when choosing an initial antimicrobial therapy $(18,79)$. Patient-related factors should also be considered, such as age, weight, allergies, and comorbidities. The presence of chronic organ dysfunction (renal or hepatic dysfunction), immunosuppressive therapy, and recent exposure to antimicrobials due to infection or colonization must be addressed. Also, it is crucial to determine the risk of MDR pathogens: prior antibiotic exposure, prolonged hospital length of stay, recent hospitalization, presence of invasive devices, local ecology and resistance patterns, and previous colonization by resistant pathogens (surveillance cultures) (18).

Different interventions have been proposed to improve empiric antimicrobial therapy in patients with sepsis. Computerized clinical decision support systems (CCDSSs) or automated antibiotic alerts, infectious diseases consultation, and local antibiotic prescription guidelines are some examples $(67,68,80)$. Recommended empirical regimens of antibiotics should be developed and continuously updated as part of local protocols in every health care institution, in order to facilitate empiric antimicrobial prescription practices, based on current practice guidelines and incorporating local and national ecology/resistance patterns (68).

\section{Combination antibiotic therapy in sepsis and septic shock}

In the light of the increasing frequency of MDR pathogens, combination antibiotic therapy is frequently recommended to ensure broader antimicrobial spectrum and adequate empiric coverage. Combination therapy is defined as the use of two different classes of antibiotics with activity against a single pathogen, mainly for two reasons: to accelerate pathogen clearance and to warrant pathogen susceptibility to treatment. Conversely, the main reasons for choosing monotherapy are reducing antibiotic pressure, the 
appearance of new infections (superinfections), antibiotic antagonism, reduced toxicity, and costs (81). The 2016 SSC guidelines suggest an empiric combination therapy for the initial management of septic shock (weak recommendation). However, the guidelines disclose a recommendation against the routine prescription of combination therapy in patients without shock or bacteremia (7). Further, a consensus committee of international experts recently reported the research priorities for sepsis and septic shock and acknowledged the lack of evidence on empiric antibiotic combination therapy in sepsis or septic shock (82).

Studies on the success of combination therapy have found mixed results, and there is a lack of well-powered randomized controlled trials assessing this issue. Several observational studies showed the superiority of combination therapy over monotherapy in patients with sepsis and septic shock (83-87). Besides, a propensity-matched analysis and a meta-analytic/ meta-regression study showed an association of combination therapy with higher survival of the most severely ill patients, who are affected by life-threatening infections with highrisk of death. However, increased mortality was observed in patients with low risk of death $(88,89)$. A randomized, parallel-group trial of 600 patients with sepsis or septic shock found no superiority of combination treatment over monotherapy in organ failure or mortality between the two groups (90). In a meta-analysis of monotherapy versus beta-lactam-aminoglycoside combination therapy in sepsis, Paul et al. found no differences in mortality. Higher rates of side effects (mainly nephrotoxicity) were reported in the combination group (91). In a setting in which local ecology and resistance patterns showed a low prevalence of MDR strains, a short course of empirical adjunctive gentamicin was associated with an increased incidence of acute renal failure, without a benefit in survival (92).

The IDSA supports the empiric use of two active agents against gram-negative bacilli for the empiric treatment of septic shock, to increase the chance of having at least one active agent against the potential pathogen involved (56). Recognizing the requirement to promote the judicious use of antibiotics, the IDSA convened a workgroup to address recommendations for appropriate antibiotic use for the treatment of sepsis. For a total of 21 antibiotic class combinations, ten were approved by the experts. Concerns regarding increasing resistance and adequate coverage of pathogens were cited as the reasons to choose such combinations. Among the most important recommendations, any combination with macrolides or ciprofloxacin, as well as the specific combinations of aztreonam with cephalosporins and aminoglycosides with intravenous clindamycin were not approved for the empiric treatment of sepsis (93).

Based on contradictory evidence, some experts support using two antibiotics for the empiric treatment of patients with septic shock and upon suspicion of infection with a MDR pathogen. Nevertheless, after microbiological isolation or favorable clinical response, treatment can be narrowed into a tailored therapy of the shortest acceptable duration, even with negative culture results (94). Wellpowered randomized controlled trials evaluating different antibiotic combinations, and performed in different settings should be conducted, to determine whether any specific combination is superior over the others (82).

\section{De-escalation of antimicrobial therapy}

Narrowing empiric therapy to a tailored therapy is considered essential for a successful antibiotic stewardship program. De-escalation programs are focused on reducing antimicrobial resistance and adverse drug-related events. The 2016 SSC guidelines recommend that the antibiotic spectrum should be narrowed when causing pathogens and their antibiotic susceptibility are known, or when a favorable clinical course is present (7).

There are several observational studies, and a meta-analysis including a high number of patients, that have found deescalation therapy to be safe practice associated with lower mortality rates in patients with severe sepsis and septic shock (95-98). In an observational study of oncologic patients, Mokart et al. reported that de-escalation was performed in $40 \%$ of neutropenic patients; however, it had no adverse impact on mortality (99). In this study, both the appropriateness of empiric antibiotic treatment and compliance with clinical guidelines were independently associated with the frequency of de-escalation. In a randomized controlled trial, Leone $e t$ al. evaluated de-escalation in patients with sepsis. In this study of 116 patients, the hospital length of stay was significantly longer in the de-escalation group, though it did not affect the mortality rate (100).

Even though antibiotic de-escalation has been recognized as a safe practice, its implementation has been widely variable among different settings (101). It is estimated that both in the United States and Europe, only $11 \%$ to $55 \%$ of treatments are de-escalated (102). In a previous study of patients with ventilator-associated and pneumonia (VAP), Niederman and Soulountsi evaluated the implementation of a de-escalation strategy. In such study, the compliance was variable (of around $23 \%$ to $74 \%$ ) among participants, 
and the main barriers for de-escalation were: fear of patient deterioration, fear of patients being colonized by other microorganisms different from those initially isolated, facing management strugglings when obtaining negative culture results and the lack of local guidelines for deescalation (103).

Different tools have been developed in order to promote de-escalation, in an attempt to eliminate those concerns that hinder clinicians from implementing the best available scientific evidence. Some of them include educational programs to promote de-escalation among professionals, novel microorganism identification techniques, and the use of biomarkers to guide decisions. Several initiatives promoting streamlining of antimicrobial therapy have been implemented to increase the frequency of antibiotic deescalation $(65,104)$. In the ABISS-Edusepsis study, which included 2628 adult patients in 72 ICUs, the proportion of patients who had de-escalation increased significantly from $16.3 \%$ to $20.1 \%$ after an educational intervention consisting of a multifaceted educational program on sepsis care (65).

\section{Laboratory workup}

Appropriate collection of cultures, from blood and the site of infection, is essential. Blood cultures remain to be the gold standard for the detection of bacteremia, and the 2016 SSC guidelines recommend that appropriate microbiologic cultures should be obtained before starting antimicrobial therapy (7). Pathogen detection allows antibiotic treatment to be optimized, and sterilization of cultures can occur early after the first dose of antimicrobial therapy. Sometimes, obtaining blood cultures may delay antibiotic therapy and adversely impact on patient outcomes. A prospective study evaluated the sensitivity of blood cultures obtained shortly after the initiation of antimicrobial therapy (105). The findings of this study reinforce the recommendation of taking blood cultures before starting antibiotic therapy. When cultures cannot be obtained promptly, the decision to prioritize the rapid administration of antimicrobials over taking cultures should be individualized.

The latency period between sample withdrawal and culture results may be too long. In addition, obtaining adequate samples may be difficult, and there is a significant proportion of patients with negative results. Novel technologies, such as the matrix-assisted laser desorption ionization-time of flight (MALDI-TOF), mass spectrometry, and PCR-assays, may improve appropriateness of antimicrobial therapy, by reducing time to directed therapy and identifying resistant organisms rapidly. However, these methods are not widely available and require an effort to translate improvements into fast and adequate actions taken by clinicians. Of note, these rapid diagnostic tests must be interpreted with caution and should be combined with educational and AS programs. The next-generation sequencing (NGS) technique, also known as high-throughput sequencing, is a highly promising method for pathogen identification. This technology allows for sequencing of DNA and RNA of all likely pathogens much more quickly and cheaply than the previously used Sanger sequencing and will help to predict particular resistance phenotypes. This method is not widely implemented in clinical practice (106).

\section{Duration of antimicrobial therapy}

Prolonged duration of antimicrobial therapy is associated with increased resistance, higher risk of drug-associated adverse effects, and higher costs. Disregarding special situations (e.g., infective endocarditis, gram-negative meningitis, osteomyelitis, fungal infections), prolonged antimicrobial therapy has not been demonstrated to be beneficial. Reducing the duration of antibiotic treatment is the most effective strategy to reduce antibiotic resistance: by reducing the pressure on the endogenous flora and selection of resistant strains (102).

Several clinical studies and meta-analyses on common infections have shown that short treatments are safer than longer treatments, without affecting treatment success (107-110). In a review article on the duration of antimicrobial therapy in critically ill patients, Zilahi et al. concluded that shorter antibiotic courses were effective and safe, although they did not recommend a "one size fits all" approach for all situations and specifies a set of recommendations (111). Pugh et al. compared 8-vs. 5-day courses of antibiotic therapy for VAP. Shorter courses of therapy increased antibiotic-free days and reduced VAP infections caused by MDR microorganisms, while not adversely affecting mortality or treatment failure rates (112). Chotiprasitsakul et al. demonstrated that shorter (6-10 days) versus extended regimens (11-16 days) for Enterobacteriaceae bacteremia had similar results, while a protective effect against MDR gram-negative bacteria was observed (113). Short courses of treatment for invasive meningococcal disease (4- vs. 7 -day courses) were also successful without worsening the risk of infection recurrences (114). In a trial of 539 patients with intra-abdominal infection, Sawyer et al. evaluated a predetermined length of antibiotic therapy versus standard 
practice for antimicrobial treatment of intra-abdominal infections (108). In patients with effective source control, there was no difference in outcomes comparing a 4-day course to usual care (8 days). In a prospective randomized trial on VAP, Chastre et al. compared 8 to 15 days of therapy (107). Patients receiving shorter courses of therapy were less prone to have resistant pathogens in recurrent pulmonary infections, and there was no difference in mortality or length of ICU stay.

Nevertheless, in different circumstances, the duration of therapy cannot be well-defined, as adjustments of antimicrobial prescription mainly rely on effective source control (115). Although initiation of antibiotics may represent a difficult decision, stopping antibiotic therapy often proves to be even more difficult in some particular situations. Intra-abdominal infections or infected necrotizing pancreatitis often present a complicated clinical course. Effective control of infection cannot be timely achieved in some cases, favoring the emergence of antimicrobial resistance. Decisions on duration of antibiotic therapy need to be individualized, being necessary to consider different patient-related factors (e.g., severity of illness, clinical response), the type of infection (e.g., source control, deep-seated infection, MDR pathogens) and the availability of diagnostic tools (e.g., clinical/laboratory scores, biomarkers) (81).

\section{Biomarkers to guide antimicrobial therapy in sepsis}

Currently, several biomarkers are used for diagnosis, treatment management, and prognosis estimation in sepsis. C-reactive protein (CRP) and procalcitonin (PCT) are the most broadly studied. PCT is more specific than CRP for bacterial infections, and its use has been proposed to avoid the unnecessary use of antibiotics (116). The halflife of PCT is around $\sim 24$ hours, and a progressive decrease in plasma levels is associated with effective antibiotic therapy. Several studies have evaluated the contribution of algorithms guided by PCT plasma levels to reduce the duration of antibiotic treatments without adversely affecting clinical outcomes (117).

The beneficial effects of PCT-based algorithms have been demonstrated in critically ill patients. However, in patients with lower respiratory tract infections, the PCTguided approach may not be adequate (118). In the case of sepsis and septic shock, PCT levels of $<0.5 \mathrm{ng} / \mathrm{mL}$ or a decrease of $80 \%$ of the highest PCT peak concentration levels have been observed to be useful to support the clinical decision of stopping antibiotics while also predicting favorable outcomes $(119,120)$.

As it can be affected by other infection-like inflammatory conditions, PCT is a non-specific biomarker of infection, making its interpretation challenging when treating surgical patients. A PCT-based algorithm was not observed to be useful in a single-center study, including patients with intraabdominal infection and septic shock (121). Garcia et al. demonstrated that PCT was useful to diagnose bacterial infection in children undergoing on-pump cardiac surgery; however, a higher PCT cut-off of $2 \mathrm{ng} / \mathrm{mL}$ was used, rather than the classic cut-off for medical patients (122). CRP also plays a role in AS programs. A recent trial, including septic patients, found that CRP was as useful as PCT for reducing antibiotic use, with no associated harms (123). In a recent meta-analysis, the PCT-guided treatment algorithm demonstrated a beneficial effect in terms of reducing the duration of antibiotic treatment (7.35 vs. 8.85 days; $\mathrm{P}<0.001)$ and even a trend in reducing the length of ICU stay (11.09 vs. 11.91 days; $\mathrm{P}=0.329)$ with no adverse impact on mortality $(\mathrm{P}=0.114)(124,125)$.

There are no relevant studies on the use of PCT to support the decision to de-escalate antibiotics in critically ill patients once culture results are available; therefore, it is not reasonable to use PCT levels to reduce antibiotic spectrum. Infection biomarkers are widely extended into daily clinical practice to support antibiotic discontinuation in critically ill patients with sepsis and septic shock. The use of biomarkers provides an opportunity to improve patients' care; however, they do not substitute clinical judgment.

\section{Dose optimization-antimicrobial PK and PD}

Antibiotic efficacy is determined both by the prescription of an optimal dose and the presence of adequate tissue concentrations at the target site. Optimized antibiotic dosing regimens to ensure therapeutic levels of antimicrobials improve clinical results, minimize drug toxicity and drug-resistance, and reduce mortality in critically ill patients (125).

Critically ill patients present profound pathophysiological changes and require interventions for organ support that may produce significant alterations PK of antibiotics (126). Abnormal volume distribution (capillary leak, hypoalbuminemia), hyperdynamic circulation, and variations in renal or liver function are the most relevant and frequent pathophysiological mechanisms involved. Of note, antibiotic concentration may be altered during renal replacement 
therapy (RRT) and extracorporeal membrane oxygenation (ECMO) support techniques (127). These aspects result in significant fluctuations of antibiotic concentrations within the same patient, leading to unpredictable pharmacological and toxicological effects. Therefore, using standard doses can result in sub-optimal concentrations or even in drug accumulation leading to drug toxicity (128).

During the initial phase of sepsis, there is an increase in the distribution volume, which limits therapeutic concentrations of hydrophilic antibiotics to be reached in some tissues (129). Also, an increase in renal clearance can be associated which an increase of drug elimination by the kidneys (130-132). These alterations lead to the need for increasing the loading dose of frequently used antibiotics (beta-lactams, aminoglycosides, glycopeptides, colistin) in sepsis. In a prospective study of 236 critical patients, $50 \%$ of patients required an increase in the standard dose of betalactam antibiotics after the first monitoring of antimicrobial plasma levels (133). In a prospective multicenter study of patients with sepsis and septic shock, Taccone et al. demonstrated that conventional initial dosing for many beta-lactams was insufficient to reach the PK/PD targets on the first day (134). An initial dose of 1.5 times the standard dose was suggested as a general approach (135).

As sepsis-associated multi-organ dysfunction progresses, it generally follows a predictable course, with impaired renal and hepatic function. Therefore, maintenance doses are conditioned by the drug clearance route, and PD should be considered due to the increasing number of MDR pathogens.

The Defining Antibiotic Levels in Intensive care unit patients (DALI) study (136), a large multicenter prospective study conducted in 248 patients with severe infection treated with beta-lactam antibiotics, found that one-fifth of patients did not achieve the PK/PD targets, and 500-fold differences in antibiotic blood concentrations were observed. Even higher drug exposure was associated with favorable clinical outcomes.

Of note, it is recommended not to reduce (or even increase) standard antibiotic dosing in patients on continuous renal replacement therapy (CRRT), since there is no evidence of drug accumulation in such a scenario. Higher doses and extended infusions of antibiotics are desirable for most cases, particularly for the treatment of MDR bacteria (127). There is a paucity of data regarding antibiotic dosing in ECMO; in the meanwhile, standard dosage regimens are recommended $(81,127)$.

Several studies and a recent meta-analysis, including a RCT, found prolonged infusions of antibiotics to be associated with lower mortality than short-term infusions or intermittent-bolus dosing. The higher efficacy of betalactam antibiotics for targeting MDR microorganisms have been observed with prolonged infusions (127,137-140). Similarly, prolonged infusions are better to achieve effective PK/PD targets and reduce the toxicity of linezolid and vancomycin $(141,142)$. However, the evidence is conflicting regarding the reduction of mortality rates (143).

An individualized approach for antibiotic dosing is therefore required for patients with sepsis, and therapeutic drug monitoring (TDM) contributes to valuable information for that task. TDM has been well accepted for monitoring antibiotics associated with higher toxicity, such as aminoglycosides and glycopeptides (vancomycin). Recent evidence also suggests beta-lactam TDM as a useful strategy to optimize drug exposure (127). Roberts et al. proposed that, in the future, it is likely that highly sophisticated approaches using TDM combined with Bayesian forecasting coupled with dosing software for accurate antibiotic dosing may be applied (125).

\section{Conclusions}

Sepsis is a medical emergency with unacceptably high mortality and adverse outcomes. In this context, appropriate and timely antibiotic treatment is one of the cornerstones of therapy to prevent deaths and life-threatening complications. When treating suspicious or confirmed cases of sepsis, clinicians must ensure the administration a broad-spectrum antimicrobial within the first hour of diagnosis to reduce the risk of death. Also, optimizing antibiotic use is essential to ensure successful outcomes and to reduce antibiotic-associated harm, and the spread of antibiotic resistance. In this context, it is vital to consider AS as an essential part of sepsis management. It implies individualizing appropriate antimicrobial therapy. Hospitals should develop, and update periodically, specific empirical regimens for specific sites of infection, incorporating local and national microbiology and resistance patterns based on practice guidelines to facilitate empirical antimicrobial prescription. Appropriate collection of cultures is essential for antimicrobial sepsis management. AS also implies deescalation and shortening treatment duration. In critically ill patients, an individual dosing approach should replace the principle of standard dosing. Higher loading doses and an extended or continuous perfusion of beta-lactam are indicated for most patients. Continuous re-assessment of 
disease progression and adequate antibiotic therapy should be incorporated as part of usual care for patients with sepsis. Leadership, teamwork, AS frameworks, clinical guidelines recommendations on the optimal duration of treatments, de-escalation, PCT-based algorithms, and new diagnostic stewardship approaches will help us to improve patients' quality of care.

\section{Acknowledgments}

Funding: None.

\section{Footnote}

Conflicts of Interest: The authors have no conflicts of interest to declare.

Ethical Statement: The authors are accountable for all aspects of the work in ensuring that questions related to the accuracy or integrity of any part of the work are appropriately investigated and resolved.

Open Access Statement: This is an Open Access article distributed in accordance with the Creative Commons Attribution-NonCommercial-NoDerivs 4.0 International License (CC BY-NC-ND 4.0), which permits the noncommercial replication and distribution of the article with the strict proviso that no changes or edits are made and the original work is properly cited (including links to both the formal publication through the relevant DOI and the license). See: https://creativecommons.org/licenses/by-nc-nd/4.0/.

\section{References}

1. Prescott HC, Angus DC. Enhancing recovery from sepsis: a review. JAMA 2018;319:62-75.

2. Singer M, Deutschman CS, Seymour CW, et al. The Third International Consensus Definitions for sepsis and septic shock (sepsis-3). JAMA 2016;315:801-10.

3. Fleischmann C, Scherag A, Adhikari NK, et al. Assessment of global incidence and mortality of hospital-treated sepsis. Current estimates and limitations. Am J Respir Crit Care Med 2016;193:259-72.

4. Sprung CL, Annane D, Keh D, et al. Hydrocortisone therapy for patients with septic shock. N Engl J Med 2008;358:111-24.

5. Ranieri VM, Thompson BT, Barie PS, et al. Drotrecogin alfa (activated) in adults with septic shock. N Engl J Med
2012;366:2055-64.

6. Plata-Menchaca EP, Ferrer R. Life-support tools for improving performance of the Surviving Sepsis Campaign Hour-1 bundle. Med Intensiva 2018;42:547-50.

7. Rhodes A, Evans LE, Alhazzani W, et al. Surviving Sepsis Campaign: international guidelines for management of sepsis and septic shock: 2016. Intensive Care Med 2017;43:304-77.

8. Levy MM, Evans LE, Rhodes A. The Surviving Sepsis Campaign Bundle: 2018 update. Intensive Care Med 2018;44:925-8.

9. Ferrer R, Martin-Loeches I, Phillips G, et al. Empiric antibiotic treatment reduces mortality in severe sepsis and septic shock from the first hour: results from a guidelinebased performance improvement program. Crit Care Med 2014;42:1749-55.

10. Liu VX, Fielding-Singh V, Greene JD, et al. The timing of early antibiotics and hospital mortality in sepsis. Am J Respir Crit Care Med 2017;196:856-63.

11. Kumar A, Roberts D, Wood KE, et al. Duration of hypotension before initiation of effective antimicrobial therapy is the critical determinant of survival in human septic shock. Crit Care Med 2006;34:1589-96.

12. Kumar A, Ellis P, Arabi Y, et al. Initiation of inappropriate antimicrobial therapy results in a fivefold reduction of survival in human septic shock. Chest 2009;136:1237-48.

13. Singer M. Antibiotics for sepsis: does each hour really count, or is it incestuous amplification? Am J Respir Crit Care Med 2017;196:800-2.

14. Bernhard M, Lichtenstern C, Eckmann C, et al. The early antibiotic therapy in septic patients--milestone or sticking point? Crit Care 2014;18:671.

15. Mi MY, Klompas M, Evans L. Early administration of antibiotics for suspected sepsis. N Engl J Med 2019;380:593-6.

16. Beumier M, Casu GS, Hites M, et al. Elevated -lactam concentrations associated with neurological deterioration in ICU septic patients. Minerva Anestesiol 2015;81:497-506.

17. O’Neill J. Antimicrobial Resistance: tackling a Crisis for the Health and Wealth of Nations. 2014. Available online: https://bit.ly/36u4nKB

18. Ulldemolins M, Nuvials X, Palomar M, et al. Appropriateness is critical. Crit Care Clin 2011;27:35-51.

19. Gerding DN. The search for good antimicrobial stewardship. Jt Comm J Qual Improv 2001;27:403-4.

20. Joseph J, Rodvold KA. The role of carbapenems in the treatment of severe nosocomial respiratory tract infections. Expert Opin Pharmacother 2008;9:561-75. 
21. Barochia AV, Cui X, Vitberg D, et al. Bundled care for septic shock: an analysis of clinical trials. Crit Care Med 2010;38:668-78.

22. Levy MM, Rhodes A, Phillips GS, et al. Surviving Sepsis Campaign: association between performance metrics and outcomes in a 7.5-year study. Crit Care Med 2015;43:3-12.

23. Coz Yataco A, Jaehne AK, Rivers EP. Protocolized early sepsis care is not only helpful for patients: it prevents medical errors. Crit Care Med 2017;45:464-72.

24. Centers for Medicare \& Medicaid Services. Fact sheet: CMS to improve quality of care during hospital inpatient stays. 2014. Available online: https://www.cms.gov/ Newsroom/MediaReleaseDatabase/Fact-sheets/2014Fact-sheets-items/2014-08-04-2.html

25. Dwyer J. One Boy's Death moves state to action to prevent others. The New York Times Company. 2012. Available online: http://www.nytimes.com/2012/12/21/ nyregion/one-boys-death-moves-state-to-action-toprevent-others.html

26. Buchman TG, Azoulay E. Practice guidelines as implementation science: the journal editors' perspective. Intensive Care Med 2017;43:378-9.

27. Garey KW, Rege M, Pai MP, et al. Time to initiation of fluconazole therapy impacts mortality in patients with candidemia: a multi-institutional study. Clin Infect Dis 2006;43:25-31.

28. Bodey GP, Jadeja L, Elting L. Pseudomonas bacteremia. Retrospective analysis of 410 episodes. Arch Intern Med 1985;145:1621-9.

29. Garnacho-Montero J, Garcia-Cabrera E, Diaz-Martin A, et al. Determinants of outcome in patients with bacteraemic pneumococcal pneumonia: importance of early adequate treatment. Scand J Infect Dis 2010;42:185-92.

30. Gacouin A, Le Tulzo Y, Lavoue S, et al. Severe pneumonia due to Legionella pneumophila: prognostic factors, impact of delayed appropriate antimicrobial therapy. Intensive Care Med 2002;28:686-91.

31. Hamandi B, Holbrook AM, Humar A, et al. Delay of adequate empiric antibiotic therapy is associated with increased mortality among solid-organ transplant patients. Am J Transplant 2009;9:1657-65.

32. Harmankaya M, Oreskov JO, Burcharth J, et al. The impact of timing of antibiotics on in-hospital outcomes after major emergency abdominal surgery. Eur J Trauma Emerg Surg 2020;46:221-7.

33. Gaieski DF, Mikkelsen ME, Band RA, et al. Impact of time to antibiotics on survival in patients with severe sepsis or septic shock in whom early goal-directed therapy was initiated in the emergency department. Crit Care Med 2010;38:1045-53.

34. Puskarich MA, Trzeciak S, Shapiro NI, et al. Association between timing of antibiotic administration and mortality from septic shock in patients treated with a quantitative resuscitation protocol. Crit Care Med 2011;39:2066-71.

35. Sherwin R, Winters ME, Vilke GM, et al. Does Early and Appropriate Antibiotic Administration Improve Mortality in Emergency Department Patients with Severe Sepsis or Septic Shock? J Emerg Med 2017;53:588-95.

36. Seymour CW, Gesten F, Prescott HC, et al. Time to treatment and mortality during mandated emergency care for sepsis. N Engl J Med 2017;376:2235-44.

37. Weiss SL, Fitzgerald JC, Balamuth F, et al. Delayed antimicrobial therapy increases mortality and organ dysfunction duration in pediatric sepsis. Crit Care Med 2014;42:2409-17.

38. Whiles BB, Deis AS, Simpson SQ. Increased Time to initial antimicrobial administration is associated with progression to septic shock in severe sepsis patients. Crit Care Med 2017;45:623-9.

39. Kim RY, Ng AM, Persaud AK, et al. Antibiotic timing and outcomes in sepsis. Am J Med Sci 2018;355:524-9.

40. Peltan ID, Brown SM, Bledsoe JR, et al. ED door-toantibiotic time and long-term mortality in sepsis. Chest 2019;155:938-46.

41. Bloos F, Ruddel H, Thomas-Ruddel D, et al. Effect of a multifaceted educational intervention for anti-infectious measures on sepsis mortality: a cluster randomized trial. Intensive Care Med 2017;43:1602-12.

42. Zhang D, Micek ST, Kollef MH. Time to appropriate antibiotic therapy is an independent determinant of postinfection ICU and hospital lengths of stay in patients with sepsis. Crit Care Med 2015;43:2133-40.

43. Iscimen R, Cartin-Ceba R, Yilmaz M, et al. Risk factors for the development of acute lung injury in patients with septic shock: an observational cohort study. Crit Care Med 2008;36:1518-22.

44. Bagshaw SM, Lapinsky S, Dial S, et al. Acute kidney injury in septic shock: clinical outcomes and impact of duration of hypotension prior to initiation of antimicrobial therapy. Intensive Care Med 2009;35:871-81.

45. Garnacho-Montero J, Aldabo-Pallas T, GarnachoMontero C, et al. Timing of adequate antibiotic therapy is a greater determinant of outcome than are TNF and IL-10 polymorphisms in patients with sepsis. Crit Care 2006;10:R111.

46. Hwang SY, Shin J, Jo IJ, et al. Delayed antibiotic therapy 
and organ dysfunction in critically ill septic patients in the emergency department. J Clin Med 2019. doi: 10.3390/ jcm8020222.

47. Labelle A, Juang P, Reichley R, et al. The determinants of hospital mortality among patients with septic shock receiving appropriate initial antibiotic treatment* ${ }^{*}$ Crit Care Med 2012;40:2016-21.

48. de Groot B, Ansems A, Gerling DH, et al. The association between time to antibiotics and relevant clinical outcomes in emergency department patients with various stages of sepsis: a prospective multi-center study. Crit Care 2015;19:194.

49. van Paridon BM, Sheppard C, G GG, et al. Timing of antibiotics, volume, and vasoactive infusions in children with sepsis admitted to intensive care. Crit Care 2015;19:293.

50. Creedon JK, Vargas S, Asaro LA, et al. Timing of antibiotic administration in pediatric sepsis. Pediatr Emerg Care 2018. [Epub ahead of print].

51. Alam N, Oskam E, Stassen PM, et al. Prehospital antibiotics in the ambulance for sepsis: a multicentre, open label, randomised trial. Lancet Respir Med 2018;6:40-50.

52. Sterling SA, Miller WR, Pryor J, et al. The Impact of timing of antibiotics on outcomes in severe sepsis and septic shock: a systematic review and meta-analysis. Crit Care Med 2015;43:1907-15.

53. Johnston ANB, Park J, Doi SA, et al. Effect of immediate administration of antibiotics in patients with sepsis in tertiary care: a systematic review and meta-analysis. Clin Ther 2017;39:190-202.e6.

54. Laupland KB, Ferrer R. Is it time to implement door-toneedle time for "infection attacks"? Intensive Care Med 2017;43:1712-3

55. Kalil AC, Metersky ML, Klompas M, et al. Management of adults with hospital-acquired and ventilator-associated pneumonia: 2016 clinical practice guidelines by the Infectious Diseases Society of America and the American Thoracic Society. Clin Infect Dis 2016;63:e61-111.

56. IDSA Sepsis Task Force. Infectious Diseases Society of America (IDSA) position statement: why IDSA did not endorse the Surviving Sepsis Campaign Guidelines. Clin Infect Dis 2018;66:1631-5.

57. Klompas M, Calandra T, Singer M. Antibiotics for sepsisfinding the equilibrium. JAMA 2018;320:1433-4.

58. Marik PE, Farkas JD. The changing paradigm of sepsis: early diagnosis, early antibiotics, early pressors, and early adjuvant treatment. Crit Care Med 2018;46:1690-2 .

59. Seymour CW, Liu VX, Iwashyna TJ, et al. Assessment of clinical criteria for sepsis: for the third international consensus definitions for sepsis and septic shock (sepsis-3). JAMA 2016;315:762-74.

60. Filbin MR, Thorsen JE, Zachary TM, et al. Antibiotic delays and feasibility of a 1-hour-from-triage antibiotic requirement: analysis of an emergency department sepsis quality improvement database. Ann Emerg Med 2020;75:93-9.

61. Amaral AC, Fowler RA, Pinto R, et al. Patient and organizational factors associated with delays in antimicrobial therapy for septic shock. Crit Care Med 2016;44:2145-53.

62. Peltan ID, Mitchell KH, Rudd KE, et al. Prehospital care and emergency department door-to-antibiotic time in sepsis. Ann Am Thorac Soc 2018;15:1443-50.

63. Vattanavanit V, Buppodom T, Khwannimit B. Timing of antibiotic administration and lactate measurement in septic shock patients: a comparison between hospital wards and the emergency department. Infect Drug Resist 2018;11:125-32.

64. Petit J, Passerieux J, Maître O, et al. Impact of a qSOFAbased triage procedure on antibiotic timing in ED patients with sepsis: a prospective interventional study. Am J Emerg Med 2019. [Epub ahead of print].

65. Ferrer R, Martinez ML, Goma G, et al. Improved empirical antibiotic treatment of sepsis after an educational intervention: the ABISS-Edusepsis study. Crit Care 2018;22:167.

66. Kim M, Song KH, Kim CJ, et al. Electronic alerts with automated consultations promote appropriate antimicrobial prescriptions. PLoS One 2016;11:e0160551.

67. Micek ST, Heard KM, Gowan M, et al. Identifying critically ill patients at risk for inappropriate antibiotic therapy: a pilot study of a point-of-care decision support alert. Crit Care Med 2014;42:1832-8.

68. Barlam TF, Cosgrove SE, Abbo LM, et al. Executive summary: implementing an antibiotic stewardship program: guidelines by the Infectious Diseases Society of America and the Society for Healthcare Epidemiology of America. Clin Infect Dis 2016;62:1197-202.

69. Garnacho-Montero J, Gutierrez-Pizarraya A, EscorescaOrtega A, et al. Adequate antibiotic therapy prior to ICU admission in patients with severe sepsis and septic shock reduces hospital mortality. Crit Care 2015;19:302.

70. Walsh D, Gekle R, Bramante R, et al. Emergency department sepsis huddles: Achieving excellence for sepsis benchmarks in New York State. Am J Emerg Med 2019. [Epub ahead of print]. 
71. Hitti EA, Lewin JJ, 3rd, Lopez J, et al. Improving door-toantibiotic time in severely septic emergency department patients. J Emerg Med 2012;42:462-9.

72. Kufel WD, Seabury RW, Meola GM, et al. Impact of premix antimicrobial preparation and time to administration in septic patients. CJEM 2018;20:565-71.

73. Vallés J, Rello J, Ochagavía A, et al. Community-acquired bloodstream infection in critically ill adult patients: impact of shock and inappropriate antibiotic therapy on survival. Chest 2003;123:1615-24.

74. Suberviola Cañas B, Jáuregui R, Ballesteros MÁ, et al. Effects of antibiotic administration delay and inadequacy upon the survival of septic shock patients. Med Intensiva 2015;39:459-66.

75. Zilberberg MD, Shorr AF, Micek ST, et al. Multi-drug resistance, inappropriate initial antibiotic therapy and mortality in Gram-negative severe sepsis and septic shock: a retrospective cohort study. Crit Care 2014;18:596.

76. Vazquez-Guillamet C, Scolari M, Zilberberg MD, et al. Using the number needed to treat to assess appropriate antimicrobial therapy as a determinant of outcome in severe sepsis and septic shock. Crit Care Med 2014;42:2342-9.

77. Garnacho-Montero J, Garcia-Garmendia JL, BarreroAlmodovar A, et al. Impact of adequate empirical antibiotic therapy on the outcome of patients admitted to the intensive care unit with sepsis. Crit Care Med 2003;31:2742-51.

78. Battleman DS, Callahan M, Thaler HT. Rapid antibiotic delivery and appropriate antibiotic selection reduce length of hospital stay of patients with community-acquired pneumonia: link between quality of care and resource utilization. Arch Intern Med 2002;162:682-8.

79. Green RS, Gorman SK. Emergency department antimicrobial considerations in severe sepsis. Emerg Med Clin North Am 2014;32:835-49.

80. Pogue JM, Mynatt RP, Marchaim D, et al. Automated alerts coupled with antimicrobial stewardship intervention lead to decreases in length of stay in patients with gramnegative bacteremia. Infect Control Hosp Epidemiol 2014;35:132-8.

81. Vincent JL, Bassetti M, Francois B, et al. Advances in antibiotic therapy in the critically ill. Crit Care 2016;20:133.

82. Coopersmith CM, De Backer D, Deutschman CS, et al. Surviving Sepsis Campaign: research priorities for sepsis and septic shock. Crit Care Med 2018;46:1334-56.

83. Díaz-Martín A, Martínez-González ML, Ferrer R, et al. Antibiotic prescription patterns in the empiric therapy of severe sepsis: combination of antimicrobials with different mechanisms of action reduces mortality. Crit Care 2012;16:R223.

84. Martin-Loeches I, Lisboa T, Rodriguez A, et al. Combination antibiotic therapy with macrolides improves survival in intubated patients with community-acquired pneumonia. Intensive Care Med 2010;36:612-20.

85. Al-Hasan MN, Wilson JW, Lahr BD, et al. Beta-lactam and fluoroquinolone combination antibiotic therapy for bacteremia caused by gram-negative bacilli. Antimicrob Agents Chemother 2009;53:1386-94.

86. Delannoy PY, Boussekey N, Devos P, et al. Impact of combination therapy with aminoglycosides on the outcome of ICU-acquired bacteraemias. Eur J Clin Microbiol Infect Dis 2012;31:2293-9.

87. Micek ST, Welch EC, Khan J, et al. Empiric combination antibiotic therapy is associated with improved outcome against sepsis due to Gram-negative bacteria: a retrospective analysis. Antimicrob Agents Chemother 2010;54:1742-8.

88. Kumar A, Safdar N, Kethireddy S, et al. A survival benefit of combination antibiotic therapy for serious infections associated with sepsis and septic shock is contingent only on the risk of death: a meta-analytic/meta-regression study. Crit Care Med 2010;38:1651-64.

89. Kumar A, Zarychanski R, Light B, et al. Early combination antibiotic therapy yields improved survival compared with monotherapy in septic shock: a propensity-matched analysis. Crit Care Med 2010;38:1773-85.

90. Brunkhorst FM, Oppert M, Marx G, et al. Effect of empirical treatment with moxifloxacin and meropenem vs meropenem on sepsis-related organ dysfunction in patients with severe sepsis: a randomized trial. JAMA 2012;307:2390-9.

91. Paul M, Lador A, Grozinsky-Glasberg S, et al. Beta lactam antibiotic monotherapy versus beta lactam-aminoglycoside antibiotic combination therapy for sepsis. Cochrane Database Syst Rev 2014;(1):CD003344.

92. Ong DSY, Frencken JF, Klein Klouwenberg PMC, et al. Short-course adjunctive gentamicin as empirical therapy in patients with severe sepsis and septic shock: a prospective observational cohort study. Clin Infect Dis 2017;64:1731-6.

93. Septimus EJ, Coopersmith CM, Whittle J, et al. Sepsis national hospital inpatient quality measure (SEP-1): multistakeholder work group recommendations for appropriate antibiotics for the treatment of sepsis. Clin Infect Dis 2017;65:1565-9.

94. Klompas M. Monotherapy is adequate for septic shock due to gram-negative organisms. Crit Care Med 2017;45:1930-2. 
95. Turza KC, Politano AD, Rosenberger LH, et al. De-escalation of antibiotics does not increase mortality in critically ill surgical patients. Surg Infect (Larchmt) 2016;17:48-52.

96. Garnacho-Montero J, Gutierrez-Pizarraya A, EscorescaOrtega A, et al. De-escalation of empirical therapy is associated with lower mortality in patients with severe sepsis and septic shock. Intensive Care Med 2014;40:32-40.

97. Tabah A, Cotta MO, Garnacho-Montero J, et al. A Systematic review of the definitions, determinants, and clinical outcomes of antimicrobial de-escalation in the intensive care unit. Clin Infect Dis 2016;62:1009-17.

98. Gutiérrez-Pizarraya A, Leone M, Garnacho-Montero $\mathrm{J}$, et al. Collaborative approach of individual participant data of prospective studies of de-escalation in nonimmunosuppressed critically ill patients with sepsis. Expert Rev Clin Pharmacol 2017;10:457-65.

99. Mokart D, Slehofer G, Lambert J, et al. De-escalation of antimicrobial treatment in neutropenic patients with severe sepsis: results from an observational study. Intensive Care Med 2014;40:41-9.

100.Leone M, Bechis C, Baumstarck K, et al. De-escalation versus continuation of empirical antimicrobial treatment in severe sepsis: a multicenter non-blinded randomized noninferiority trial. Intensive Care Med 2014;40:1399-408.

101.van den Bosch CM, Hulscher ME, Natsch S, et al. Development of quality indicators for antimicrobial treatment in adults with sepsis. BMC Infect Dis 2014;14:345.

102. Hayashi Y, Paterson DL. Strategies for reduction in duration of antibiotic use in hospitalized patients. Clin Infect Dis 2011;52:1232-40.

103. Niederman MS, Soulountsi V. De-escalation therapy: is it valuable for the management of ventilator-associated pneumonia? Clin Chest Med 2011;32:517-34.

104. Esteban E, Belda S, Garcia-Soler P, et al. A multifaceted educational intervention shortened time to antibiotic administration in children with severe sepsis and septic shock: ABISS Edusepsis pediatric study. Intensive Care Med 2017;43:1916-8.

105. Cheng MP, Stenstrom R, Paquette K, et al. Blood culture results before and after antimicrobial administration in patients with severe manifestations of sepsis: a diagnostic study. Ann Intern Med 2019. [Epub ahead of print].

106. Richter DC, Heininger A, Brenner T, et al. Bacterial sepsis: diagnostics and calculated antibiotic therapy. Anaesthesist 2019;68:40-62.

107. Chastre J, Wolff M, Fagon JY, et al. Comparison of 8 vs 15 days of antibiotic therapy for ventilator-associated pneumonia in adults: a randomized trial. JAMA
2003;290:2588-98.

108. Sawyer RG, Claridge JA, Nathens AB, et al. Trial of shortcourse antimicrobial therapy for intraabdominal infection. N Engl J Med 2015;372:1996-2005.

109. Hepburn MJ, Dooley DP, Skidmore PJ, et al. Comparison of short-course (5 days) and standard (10 days) treatment for uncomplicated cellulitis. Arch Intern Med 2004;164:1669-74.

110.el Moussaoui R, de Borgie CA, van den Broek P, et al. Effectiveness of discontinuing antibiotic treatment after three days versus eight days in mild to moderate-severe community acquired pneumonia: randomised, double blind study. BMJ 2006;332:1355.

111.Zilahi G, McMahon MA, Povoa P, et al. Duration of antibiotic therapy in the intensive care unit. J Thorac Dis 2016;8:3774-80.

112.Pugh R, Grant C, Cooke RP, et al. Short-course versus prolonged-course antibiotic therapy for hospital-acquired pneumonia in critically ill adults. Cochrane Database Syst Rev 2015;(8):CD007577.

113. Chotiprasitsakul D, Han JH, Cosgrove SE, et al. Comparing the outcomes of adults with Enterobacteriaceae bacteremia receiving short-course versus prolonged-course antibiotic therapy in a multicenter, propensity scorematched cohort. Clin Infect Dis 2018;66:172-7.

114. Cabellos C, Pelegrin I, Benavent E, et al. Invasive meningococcal disease: impact of short course therapy. A DOOR/RADAR study. J Infect 2017;75:420-5.

115.Martínez ML, Ferrer R, Torrents E, et al. Impact of source control in patients with severe sepsis and septic shock. Crit Care Med 2017;45:11-9.

116. Saeed K, Gonzalez Del Castillo J, Backous C, et al. Hot topics on procalcitonin use in clinical practice, can it help antibiotic stewardship? Int J Antimicrob Agents 2019;54:686-96.

117. de Jong E, van Oers JA, Beishuizen A, et al. Efficacy and safety of procalcitonin guidance in reducing the duration of antibiotic treatment in critically ill patients: a randomised, controlled, open-label trial. Lancet Infect Dis 2016;16:819-27.

118.Huang DT, Yealy DM, Filbin MR, et al. Procalcitoninguided use of antibiotics for lower respiratory tract infection. N Engl J Med 2018;379:236-49.

119. Bouadma L, Luyt CE, Tubach F, et al. Use of procalcitonin to reduce patients' exposure to antibiotics in intensive care units (PRORATA trial): a multicentre randomised controlled trial. Lancet 2010;375:463-74.

120.Sager R, Kutz A, Mueller B, et al. Procalcitonin-guided diagnosis and antibiotic stewardship revisited. BMC Med 
2017;15:15.

121.Jung B, Molinari N, Nasri M, et al. Procalcitonin biomarker kinetics fails to predict treatment response in perioperative abdominal infection with septic shock. Crit Care 2013;17:R255.

122. Garcia IJ, Gargallo MB, Torne EE, et al. Procalcitonin: a useful biomarker to discriminate infection after cardiopulmonary bypass in children. Pediatr Crit Care Med 2012;13:441-5.

123. Oliveira CF, Botoni FA, Oliveira CR, et al. Procalcitonin versus $\mathrm{C}$-reactive protein for guiding antibiotic therapy in sepsis: a randomized trial. Crit Care Med 2013;41:2336-43.

124. Iankova I, Thompson-Leduc P, Kirson NY, et al. Efficacy and safety of procalcitonin guidance in patients with suspected or confirmed sepsis: a systematic review and meta-analysis. Crit Care Med 2018;46:691-8.

125. Roberts JA, Roger C, De Waele JJ. Personalized antibiotic dosing for the critically ill. Intensive Care Med 2019;45:715-8.

126. Ulldemolins M, Roberts JA, Lipman J, et al. Antibiotic dosing in multiple organ dysfunction syndrome. Chest 2011;139:1210-20.

127. Veiga RP, Paiva JA. Pharmacokinetics-pharmacodynamics issues relevant for the clinical use of beta-lactam antibiotics in critically ill patients. Crit Care 2018;22:233.

128. Roberts JA, Taccone FS, Lipman J. Understanding PK/ PD. Intensive Care Med 2016;42:1797-800.

129. Roberts JA, Lipman J. Antibacterial dosing in intensive care: pharmacokinetics, degree of disease and pharmacodynamics of sepsis. Clin Pharmacokinet 2006;45:755-73.

130. Udy AA, Lipman J, Jarrett P, et al. Are standard doses of piperacillin sufficient for critically ill patients with augmented creatinine clearance? Crit Care 2015;19:28.

131.Huttner A, Von Dach E, Renzoni A, et al. Augmented renal clearance, low beta-lactam concentrations and clinical outcomes in the critically ill: an observational prospective cohort study. Int J Antimicrob Agents 2015;45:385-92.

132. Carlier M, Carrette S, Roberts JA, et al. Meropenem and piperacillin/tazobactam prescribing in critically ill patients: does augmented renal clearance affect pharmacokinetic/ pharmacodynamic target attainment when extended infusions are used? Crit Care 2013;17:R84.

133. Roberts JA, Ulldemolins M, Roberts MS, et al.

Therapeutic drug monitoring of beta-lactams in critically ill patients: proof of concept. Int J Antimicrob Agents 2010;36:332-9.

134. Taccone FS, Laterre PF, Dugernier T, et al. Insufficient beta-lactam concentrations in the early phase of severe sepsis and septic shock. Crit Care 2010;14:R126.

135. De Backer D, Cecconi M, Lipman J, et al. Challenges in the management of septic shock: a narrative review. Intensive Care Med 2019;45:420-33.

136. Roberts JA, Paul SK, Akova M, et al. DALI: defining antibiotic levels in intensive care unit patients: are current beta-lactam antibiotic doses sufficient for critically ill patients? Clin Infect Dis 2014;58:1072-83.

137.Abdul-Aziz MH, Lipman J, Akova M, et al. Is prolonged infusion of piperacillin/tazobactam and meropenem in critically ill patients associated with improved pharmacokinetic/pharmacodynamic and patient outcomes? An observation from the defining antibiotic levels in intensive care unit patients (DALI) cohort. J Antimicrob Chemother 2016;71:196-207.

138. Falagas ME, Tansarli GS, Ikawa K, et al. Clinical outcomes with extended or continuous versus short-term intravenous infusion of carbapenems and piperacillin/tazobactam: a systematic review and meta-analysis. Clin Infect Dis 2013;56:272-82.

139. Roberts JA, Abdul-Aziz MH, Davis JS, et al. Continuous versus intermittent beta-lactam infusion in severe sepsis. A meta-analysis of individual patient data from randomized trials. Am J Respir Crit Care Med 2016;194:681-91.

140. Vardakas KZ, Voulgaris GL, Maliaros A, et al. Prolonged versus short-term intravenous infusion of antipseudomonal beta-lactams for patients with sepsis: a systematic review and meta-analysis of randomised trials. Lancet Infect Dis 2018;18:108-20.

141. Hanrahan T, Whitehouse T, Lipman J, et al. Vancomycinassociated nephrotoxicity: A meta-analysis of administration by continuous versus intermittent infusion. Int J Antimicrob Agents 2015;46:249-53.

142. Taubert M, Zander J, Frechen S, et al. Optimization of linezolid therapy in the critically ill: the effect of adjusted infusion regimens. J Antimicrob Chemother 2017;72:2304-10.

143. Dulhunty JM, Roberts JA, Davis JS, et al. A multicenter randomized trial of continuous versus intermittent betalactam infusion in severe sepsis. Am J Respir Crit Care Med 2015;192:1298-305.

Cite this article as: Martínez ML, Plata-Menchaca EP, RuizRodríguez JC, Ferrer R. An approach to antibiotic treatment in patients with sepsis. J Thorac Dis 2020;12(3):1007-1021. doi: $10.21037 /$ jtd.2020.01.47 